\title{
Epidemiology, Management, and Functional Outcomes of Traumatic Brain Injury in Sub- Saharan Africa
}

\section{Jessica Eaton ${ }^{1}$, Asma Bilal Hanif, Joanna Grudziak ${ }^{3}$, Anthony Charles ${ }^{1-3}$}

BACKGROUND: Trauma accounts for 4.7 million deaths each year, with an estimated $90 \%$ of these occurring in low- and middle-income countries (LMICs). Approximately half of trauma-related deaths are caused by central nervous system injury. Because a thorough understanding of traumatic brain injury (TBI) in LMICs is essential to mitigate TBI-related mortality, we established a clinical and radiographic database to characterize $\mathrm{TBI}$ in our low-income setting.

METHODS: This is a review of prospectively collected data from Kamuzu Central Hospital, a tertiary care center in the capital of Malawi. All patients admitted from October 2016 through May 2017 with a history of head trauma, altered consciousness, and/or radiographic evidence TBI were included. We performed descriptive statistics, a Cox regression analysis, and a survival analysis.

RESULTS: There were 280 patients who met inclusion criteria; of these, $\mathbf{8 0 . 5 \%}$ were men. The mean age was $\mathbf{2 8 . 8}$ \pm 16.3 years. Median Glasgow Coma Scale (GCS) score was 12 (interquartile range, 8-15). Road traffic crashes constituted the most common injury mechanism (60.7\%). There were 148 (52.3\%) patients who received a computed tomography scan, with the most common findings being contusions $(26.1 \%)$. Of the patients, $88(33.0 \%)$ had severe $\mathrm{TBI}$, defined as a GCS score $\leq 8$, of whom $27.6 \%$ were intubated and $10.3 \%$ received tracheostomies. Overall mortality was $30.9 \%$. Of patients who survived, $80.1 \%$ made a good recovery. Female sex was protective, and the only significant predictor of poor functional outcome was

\section{Key words}

- Functional outcomes

- Low-income settings

- Trauma

- Traumatic brain injury

\section{Abbreviations and Acronyms}

CT: Computed tomography

GCS: Glasgow Coma Scale

GOS: Glasgow Outcome Scale

ICP: Intracranial pressure

ICU: Intensive care unit

KCH: Kamuzu Central Hospital presence of severe TBI (hazard ratio, 2.98; 95\% confidence interval, 1.79-4.95).

CONCLUSIONS: TBI represents a significant part of the global neurosurgical burden of disease. Implementation of proven in-hospital interventions for these patients is critical to attenuate TBI-related morbidity and mortality.

\section{INTRODUCTION}

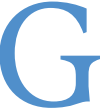
lobally, trauma is a significant cause of morbidity and mortality, with more than 4.7 million deaths and approximately $40-50$ million disabled after injury annually. ${ }^{\mathrm{I}}$ Traumatic brain injury (TBI) is the most important single injury contributing to traumatic mortality and morbidity. Injury as a whole, and TBI in particular, is expected to become a leading cause of global morbidity and mortality by the year 2020 .

In low- and middle-income countries (LMICs), where an estimated $90 \%$ of all trauma-related deaths occur, ${ }^{2}$ data suggest that up to half of all trauma-related mortality can be attributed to injury to the central nervous system. ${ }^{3}$ The odds of mortality in patients with TBI in LMICs, including sub-Saharan Africa, are more than twice as high as patients in high-income countries. ${ }^{4}$ TBI incidence ranges between 150 and 316 cases per 100,000 inhabitants per year in LMICs. ${ }^{5}$ The increasing incidence of TBI deaths is caused by a combination of urbanization, a growing middle class, the availability of cheaper cars and motorcycles, and a growing and aging population in the absence of a mature health care system. The effects of TBI are not limited to an individual's health but are also a cause of increased socioeconomic burden. ${ }^{6}$

LMIC: Low- and middle-income country

TBI: Traumatic brain injury

From the ${ }^{\mathbf{1}}$ UNC - Project Malawi, Lilongwe, Malawi; ${ }^{2}$ Department of Surgery, Kamuzu Central Hospitals, Lilongwe, Malawi; and ${ }^{3}$ Department of Surgery, University of North Carolina, Chapel Hill, North Carolina, USA

To whom correspondence should be addressed: Anthony Charles, M.D., M.P.H.

[E-mail: anthchar@med.unc.edu]

Citation: World Neurosurg. (2017) 108:650-655

https://doi.org/10.1016/j.wneu.2017.09.084

Journal homepage: www.WORLDNEUROSURGERY.org

Available online: www.sciencedirect.com 
There is little information on TBI in Africa to inform necessary public health policy, and resource-appropriate clinical management to help reduce TBI-related mortality and morbidity burden. We therefore established a TBI database at our tertiary care center in Malawi to better characterize the presentation, management, and functional outcomes after TBI in our low-resource setting.

\section{METHODS}

This is a retrospective review of prospectively collected data from Kamuzu Central Hospital (KCH). All admitted patients who presented from October 2016 through May 2017 with a history of head trauma and associated altered level of consciousness or radiographic evidence of TBI were included. Patients who were brought in dead, or treated and discharged from the emergency department, were excluded, as were patients who had head trauma but no loss of consciousness, a decrease in their Glasgow Come Scale (GCS) score, or had no radiographic evidence of TBI. Because of the retrospective nature of the study, patient consent was not obtained.

Variables collected included demographics, mechanism of injury, and vital signs. Clinical variables such as GCS score, pupillary examination, and focal neurologic findings were captured at admission and again at 24 hours after presentation. Radiographic findings and surgical interventions were recorded as they occurred throughout the hospital stay. The patient's Glasgow Outcome Scale (GOS) score, as determined by the staff neurosurgeon, was recorded at the time of patient disposition (death/ discharge/transfer/absconded).

Analyses performed include descriptive statistics of the population and a bivariate analysis in which patients were stratified according to favorable versus poor outcome, with favorable outcome being defined as good recovery or moderate disability per the GOS score, and poor outcome being defined as severe disability, vegetative state, or death. Statistical significance of differences between groups was assessed using Pearson $\chi^{2}$ test for categorical variables, analysis of variance for continuous variables, and Mann-Whitney rank-sum test for ordinal variables. Cox proportional hazards modeling and a survival time analysis, defining failure as either death or poor functional outcome (severe disability or vegetative state), were performed. Survival analysis was based on time of injury to time of discharge disposition. All analyses were performed using Stata/IC I4.I (StataCorp, College Station, Texas, USA). The University of North Carolina Institutional Review Board and the National Health Science Review Committee of Malawi approved this study.

\section{Setting}

Malawi is a land-locked country in southeastern Africa with a population of 18.6 million people, with a life expectancy of 6r.2 years and a gross domestic product of $\$ 493$ per capita. ${ }^{7} \mathrm{KCH}$ is a Iooo-bed tertiary care center, located in Lilongwe, the capital city, and serving a catchment of 6 million people in central Malawi. $\mathrm{KCH}$ has a general surgery residency and a staff of surgical consultants, including general and orthopedic surgeons, and urologists, a pediatric surgeon, and I neurosurgeon. Operating theaters are available, but operational capacity is limited by the availability of nursing and anesthetic staff. Basic laboratory investigations are available, as are x-rays, ultrasounds, and a computed tomography (CT) scanner.

\section{RESULTS}

During the 8-month study period, 280 patients were admitted to $\mathrm{KCH}$ with a primary diagnosis of TBI (Table 1). Based on data

\section{Table 1. Traumatic Brain Injury Patient Characteristics $(N=280)$}

\begin{tabular}{|c|c|}
\hline Patient Characteristics & Value \\
\hline Age, mean $\pm S D$ (years) & $28.8 \pm 16.3$ \\
\hline Male sex & $215(80.5)$ \\
\hline \multicolumn{2}{|l|}{ Injury etiology } \\
\hline RTI & $162(60.7)$ \\
\hline Assault & $66(24.7)$ \\
\hline Fall & $27(10.1)$ \\
\hline Other & $12(4.5)$ \\
\hline $\begin{array}{l}\text { Median time from injury to presentation at } \\
\mathrm{KCH} \text { (median, IQR) (days) }\end{array}$ & $0(0-1)$ \\
\hline Transferred in & $160(60.4)$ \\
\hline Admission GCS eye score (median, IQR) & $3(1-4)$ \\
\hline Admission GCS verbal score (median, IQR) & $4(2-5)$ \\
\hline Admission GCS motor score (median, IQR) & $5(4-6)$ \\
\hline Admission GCS total score (median, IQR) & $12(8-15)$ \\
\hline \multicolumn{2}{|l|}{ Neurologic signs and symptoms } \\
\hline Retrograde amnesia & $11(3.9)$ \\
\hline Weakness or paralysis & $28(10.0)$ \\
\hline Numbness & $5(1.8)$ \\
\hline Ataxia & $9(3.2)$ \\
\hline Aphasia & $18(6.4)$ \\
\hline Seizures & $27(9.6)$ \\
\hline Right pupil abnormal & $167(62.5)$ \\
\hline Left pupil abnormal & $160(60.4)$ \\
\hline Pupils asymmetrical & $64(24.6)$ \\
\hline Associated spinal injury & $9(3.2)$ \\
\hline Associated injury to chest, abdomen, and extremities & $108(40.4)$ \\
\hline \multicolumn{2}{|l|}{ Disposition from casualty } \\
\hline Admitted to ward & $179(67.6)$ \\
\hline Admitted to HDU & $25(9.4)$ \\
\hline Admitted to ICU & $21(7.9)$ \\
\hline Straight to theater & $6(2.3)$ \\
\hline Died in casualty & $34(12.8)$ \\
\hline $\begin{array}{l}\text { Values are number of patients (\%) or as otherwise indicated. } \\
\mathrm{KCH} \text {, Kamuzu Central Hospital; IQR, interquartile range; GCS, GI } \\
\text { high-dependency unit; ICU, intensive care unit; RTI, road tra }\end{array}$ & ma Scale; HDU, \\
\hline
\end{tabular}


from the $\mathrm{KCH}$ trauma database ${ }^{8}$ from 2009 to 2015 , the mean number of trauma patients admitted to $\mathrm{KCH}$ per month is 192 patients. Given our 8-month time frame, approximately ${ }_{1542}$ trauma patients were admitted during the study period, with TBI patients comprising 280 , or $18.2 \%$ of all admitted trauma patients. Most patients were men $(\mathrm{n}=2 \mathrm{I} 5,80.5 \%)$, with a mean age of $28.8 \pm \mathrm{I} 6.3$ years. Road traffic injury was the most common etiology of TBI $(n=I 62,60.7 \%)$, followed by assault $(\mathrm{n}=66,24.7 \%)$, falls $(\mathrm{n}=27$, I0.I $\%$ ), and all other etiologies $(\mathrm{n}=\mathrm{II}, 4 . \mathrm{I} \%)$. Most patients $(\mathrm{n}=\mathrm{I} 69,60.4 \%)$ were transferred to $\mathrm{KCH}$ after presenting to the nearest district hospital, and the mean time between injury and presentation to $\mathrm{KCH}$ was I. $6 \pm 6.6$ days.

\section{Clinical Findings}

At admission, the median GCS score was I2 (interquartile range, $8-\mathrm{I5}$ ). Mild TBI, defined as a GCS score of $\mathrm{I}_{3}-\mathrm{I} 5$, was seen in $\mathrm{II} 6$ patients $(43.5 \%)$, moderate TBI, defined as a GCS score of $9^{-\mathrm{I} 2}$, was seen in 65 patients $(24.3 \%)$, and severe TBI with a GCS score $\leq 8$, was seen in 88 patients $(33.0 \%)$. Sixty-four patients $(24.6 \%)$ had asymmetric pupils. On neurologic examination, the most common deficit was weakness or paralysis in at least I extremity ( $\mathrm{n}=28$, 10.0 $\%$ ), followed by aphasia $(\mathrm{n}=\mathrm{I} 8,6.4 \%)$, retrograde amnesia ( $\mathrm{n}=\mathrm{II}, 3.9 \%)$, ataxia $(\mathrm{n}=9,3.2 \%)$, and numbness $(\mathrm{n}=5, \mathrm{r} .8 \%)$. Seizures were reported in 27 patients $(9.6 \%)$. There were other concomitant injuries, to the chest, abdomen, pelvis, or extremities, in $\operatorname{lo} 8(40.4 \%)$ patients, and an associated injury to either the spinal column or spinal cord in 9 patients $(3.2 \%)$. Fiftyeight patients $(25.7 \%)$ experienced a deterioration in GCS score within 24 hours of admission.

\section{Radiologic Findings}

CT scans (Table 2) were obtained for 148 patients (52.3\%), with $56.8 \%$ of patients $(n=50)$ with severe TBI receiving scans. Of scans that were performed, I9 (I2.8\%) were normal. The most common pathology seen was brain contusion ( $\mathrm{n}=73,26 . \mathrm{\%} \%$ ), followed by skull fractures $\left(\mathrm{n}=6_{3}, 22.5 \%\right.$ ), epidural hematomas $(\mathrm{n}=2 \mathrm{I}, 7 \cdot 5 \%)$, subarachnoid hemorrhage $(\mathrm{n}=2 \mathrm{I}, 7 \cdot 5 \%)$, and subdural hematoma $(\mathrm{n}=\mathrm{I} 9,6.8 \%)$.

\section{Management}

At the time of admission, 179 patients $(67.6 \%)$ were admitted to the general ward, $25(9.4 \%)$ were admitted to the high-dependency unit, 2 I $(7.9 \%)$ were admitted to the intensive care unit (ICU), and $6(2.3 \%)$ were taken straight to the operating theater. An additional 34 patients (12.8\%) died in the casualty department. Of note, 28 patients ( $15.6 \%$ of severe TBI patients who did not die in the casualty department) with severe TBIs were admitted to the general ward.

Neck collars were placed at any time during hospitalization in $\mathrm{I} 4.3 \%$ of patients $(\mathrm{n}=40)$. Burr holes for evacuation of extra-axial hematoma, or aspiration of brain abscess in the case of patients with delayed presentation, were placed in 2I patients $(7.5 \%)$, with a postoperative mortality of 3 (I4.3\%). Mechanical ventilation was used in 25 patients $(8.9 \%)$. Of patients with severe TBI who survived beyond admission, I6 (27.6\%) received mechanical ventilation and 6 (го. $3 \%$ ) underwent tracheostomies. Eleven patients
Table 2. Radiographic Findings, Interventions, and Outcomes of All Admitted Traumatic Brain Injury Patients

\begin{tabular}{|c|c|}
\hline Findings & Number of Patients (\%) \\
\hline \multicolumn{2}{|c|}{ Radiographic findings ( $n=233 ; 148$ images) } \\
\hline Skull fracture & $63(27.0)$ \\
\hline Epidural hematoma & $21(9.0)$ \\
\hline Subdural hematoma & $19(8.2)$ \\
\hline Subarachnoid hemorrhage & $21(9.0)$ \\
\hline Contusion & 73 (31.3) \\
\hline Intraventricular hemorrhage & $4(1.7)$ \\
\hline Diffuse axonal injury & $6(2.5)$ \\
\hline Basilar skull fracture & $5(2.1)$ \\
\hline Subgaleal hematoma & $12(5.2)$ \\
\hline Midline shift & $9(3.9)$ \\
\hline \multicolumn{2}{|l|}{ Interventions $(n=280)$} \\
\hline Neck collar & $40(14.3)$ \\
\hline Tracheostomy & $12(4.3)$ \\
\hline Burr hole & $21(7.5)$ \\
\hline Sedation & 39 (13.9) \\
\hline Mechanical ventilation & $25(8.9)$ \\
\hline Elevation of skull fracture & $11(3.9)$ \\
\hline Physiotherapy & $16(5.7)$ \\
\hline \multicolumn{2}{|l|}{ Outcomes $(n=266)$} \\
\hline Discharged & 174 (65.) \\
\hline Transferred & $6(2.3)$ \\
\hline Left against medical advice & $5(1.9)$ \\
\hline Died & $82(30.9)$ \\
\hline \multicolumn{2}{|l|}{ Functional outcomes ( $\mathrm{n}=265$ ) } \\
\hline Good recovery & $148(55.9)$ \\
\hline Moderate disability & $24(9.1)$ \\
\hline Severe disability & $9(3.4)$ \\
\hline Vegetative state & $2(0.8)$ \\
\hline Death & $82(30.9)$ \\
\hline
\end{tabular}

(3.9\%) required elevation of a depressed skull fracture, and I6 $(5.7 \%)$ received inpatient physiotherapy.

\section{Outcomes}

Our overall mortality (Table 2) was 30.9\% $(\mathrm{n}=82)$. Of these, 34 patients $(4 \mathrm{I} .5 \%$ of all patients who died, or $12.8 \%$ of the total population) died while in the casualty department. When stratified by severity of TBI, $4 \mathrm{I} .4 \%(\mathrm{n}=24)$ of patients with severe TBI, compared with $31.9 \%(n=15)$ of patients with moderate TBI and $17.0 \%(n=8)$ of patients with mild TBI, died during their hospital stay. The mortality rate was $7.6 \%(n=12)$ 
for patients whose highest level of care was the general ward, $27.3 \%(\mathrm{n}=9)$ for patients whose highest level of care was the high-dependency unit, and $70.6 \%(n=24)$ for patients who received care in the ICU. Further, $69.1 \%$ of patients $\left(n=5^{6}\right)$ who died did not receive a CT scan.

Of patients that survived, $80.1 \%(n=148)$ had a good recovery with no appreciable clinical neurologic deficits, $13.1 \%(n=24)$ had a moderate disability with deficits that still allowed the patient to live independently, $4.9 \%(n=9)$ had severe disability which will require assistance with activities of daily life, and I.I\% $(n=2)$ were in a vegetative state.

A bivariate analysis comparing patients by favorable functional outcome and poor functional outcome or death was performed (Table 3). There were statistically significant differences between the 2 groups in severity of their TBI, and each component of the GCS score. A survival time analysis was done (Figure 1), which showed that female sex is protective against poor functional

Table 3. Bivariate Analysis of All Traumatic Brain Injury

Patients Stratified by Favorability of Outcome

\begin{tabular}{|c|c|c|c|}
\hline Characteristic & $\begin{array}{c}\text { Favorable } \\
\text { Outcome (Good } \\
\text { Recovery/Moderate } \\
\text { Disability) } \\
\text { (n }=172,64.9 \%)\end{array}$ & $\begin{array}{l}\text { Death or } \\
\text { Poor } \\
\text { Outcome } \\
\text { (n = 93, } \\
35.1 \% \text { ) }\end{array}$ & $P$ Value \\
\hline Age, mean \pm SD (years) & $27.5 \pm 16.3$ & $31.3 \pm 16.2$ & 0.07 \\
\hline Male sex & $139(80.8)$ & 74 (79.8) & 0.81 \\
\hline Injury etiology & & & 0.43 \\
\hline RTI & $103(59.9)$ & $57(61.3)$ & \\
\hline Assault & $39(22.7)$ & $27(29.0)$ & \\
\hline Fall & $21(12.2)$ & $6(6.5)$ & \\
\hline Other & $8(4.7)$ & $3(3.2)$ & \\
\hline \multicolumn{4}{|l|}{ TBI severity } \\
\hline Mild & $101(58.7)$ & $14(15.1)$ & $<0.001$ \\
\hline Moderate & $43(25.0)$ & $21(22.6)$ & $<0.001$ \\
\hline Severe & $28(16.3)$ & $60(64.5)$ & $<0.001$ \\
\hline $\begin{array}{l}\text { Time from injury to } \\
\text { presentation at } \mathrm{KCH} \text {, } \\
\text { mean } \pm \mathrm{SD} \text { (days) }\end{array}$ & $1.7 \pm 6.8$ & $1.6 \pm 6.9$ & 0.91 \\
\hline Transferred in & $102(59.7)$ & $57(62.0)$ & 0.72 \\
\hline Admission GCS eye score & $4(3-4)$ & $1(1-3)$ & $<0.001$ \\
\hline Admission GCS verbal score & $4(3-5)$ & $2(1-4)$ & $<0.001$ \\
\hline Admission GCS motor score & $6(5-6)$ & $4(2-5)$ & $<0.001$ \\
\hline Admission GCS total score & $14(11-15)$ & $8(5-10)$ & $<0.001$ \\
\hline $\begin{array}{l}\text { Associated injury to chest, } \\
\text { abdomen, or extremities }\end{array}$ & 65 (37.8) & $42(45.2)$ & 0.15 \\
\hline \multicolumn{4}{|c|}{$\begin{array}{l}\text { Values are number of patients (\%), median (interquartile range), or as otherwise } \\
\text { indicated. } \\
\mathrm{KCH} \text {, Kamuzu Central Hospital; GCS, Glasgow Coma Scale; RTI, road traffic injury. }\end{array}$} \\
\hline
\end{tabular}

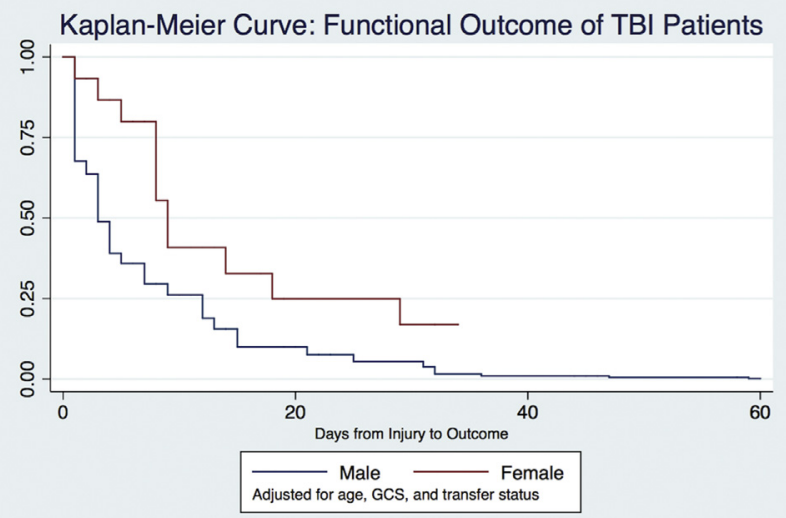

Failure based on poor functional outcomes (death, vegetative state, or severe disability)

Figure 1. Survival time analysis stratified by sex, with failure defined as death, vegetative state, or severe disability. GCS, Glasgow Coma Scale; TBI, traumatic brain injury.

outcome. On Cox regression analysis adjusted for age, sex, presence of severe TBI, and mechanism of injury (Table 4), only presence of severe TBI was a statistically significant predictor of poor outcome (hazard ratio, 2.98; 95\% confidence interval, I.79-4.95).

\section{DISCUSSION}

Most literature from sub-Saharan Africa discusses head injury, and not the more precise clinical entity of TBI. ${ }^{5}$ To our knowledge, this study is a first report of a TBI database established at a tertiary care center in sub-Saharan Africa. The prospective collection of clinical and radiographic data on all patients presenting with TBI allows us to characterize patterns and functional outcomes in TBI, in an effort to inform public health and assess interventions to reduce morbidity and mortality in this resource poor environment. In our setting, we found that the in-hospital mortality among patients presenting with TBI is $30.9 \%$.

Although TBI accounts for up to ro million hospitalizations each year worldwide, it is thought that the true incidence is likely underestimated. ${ }^{5}$ Patients with mild TBIs may never seek treatment; however, they are still subject to long-term

\section{Table 4. Adjusted Cox Regression Analysis}

\begin{tabular}{|lcc|}
\hline Variable & Hazard Ratio & $\begin{array}{c}\text { 95\% Confidence } \\
\text { Interval }\end{array}$ \\
\hline Age & 1.01 & $0.99-1.03$ \\
\hline Female sex & 0.93 & $0.49-1.75$ \\
\hline Presence of severe TBI & 2.98 & $1.79-4.95$ \\
\hline Penetrating mechanism of injury & 1.26 & $0.49-3.27$ \\
\hline TBI, traumatic brain injury. & & \\
\hline
\end{tabular}


impairments in memory or cognition, earning TBI the title of the "silent epidemic." 9 On the other end of the spectrum, patients with severe TBIs may die before reaching the hospital. An accurate assessment of the burden of TBI in sub-Saharan Africa is critical because its medical and socioeconomic impact in the continent with the youngest population is prohibitive. ${ }^{6}$ Projections of TBI for the future will be required to assess the need for longterm health care, nursing homes, and the psychosocial costs of neurocognitive impairment secondary to TBI.

In the United States, the estimated annual incidence of TBI is approximately 200 per I00,000 people, and the rate of hospitalization because of TBI has in fact decreased by approximately $50 \%$ since I980. Overall TBI mortality in the United States is estimated to be $3.3 \%{ }^{\text {IO }}$ This is in contrast with many LMICs, where incidence continues to increase in recent years ${ }^{\text {II }}$ and mortality remains high. ${ }^{4}$

The only population-based incidence report from sub-Saharan Africa comes from South Africa in I99I, reporting an approximate incidence of 3Io per I00,000 people. ${ }^{\text {I2 }}$ Unfortunately, the incidence of TBI continues to increase because more people are now able to own vehicles, and violence continues to be common in multiple regions. One modeling study predicts $6-\mathrm{I} 4$ million new TBI cases per year in Africa alone by the year 2050. ${ }^{\text {II }} \mathrm{A}$ high incidence of TBI is made worse by a disproportionately high mortality. ${ }^{4}$ Most African studies show that TBI tends to be in men, and it tends to have 2 peaks: $<$ Io years, and ages I5 $^{-}$ 24 years. ${ }^{13-17}$ African studies also report that the most common causes of TBI are road traffic crashes and violence in adults, and falls in children. ${ }^{5}$ In sub-Saharan Africa, unlike in high-income countries, there is no third peak because the average life expectancy is well below the $>65$ years of age group where the third peak is typically observed. ${ }^{5}$ We also found 2 age peaks (Figure 2); however, our 20-30 years of age peak was much more prominent than the $<$ Io years of age peak. Our most common mechanism of injury was road traffic crash in both adults and children, consistent with findings in all other settings. Globally, it is estimated that approximately $80 \%$ of TBIs are mild, $10 \%$ are moderate, and $10 \%$ are severe. ${ }^{18}$ We found our percentage of patients with mild TBIs to be significantly lower than global estimates. This is likely because of presentation bias. We suspect that there may also be a higher rate of mild TBIs at the district hospitals because only the most severe cases were referred to our center, resulting in the underreporting of patients.

TBI-related mortality data from hospital-based trauma surveillance registries in other sub-Saharan African countries reveal TBI mortality of $26 \%$ and $21 \%$ in Uganda ${ }^{15}$ and Ethiopia, ${ }^{17}$ respectively. Our reported mortality is higher than both the United States, and other low-income settings, at $31 \%$, despite the fact that most of our TBI patients presented on admission with a GCS score of $1_{3}-$ I5. Among patients with mild TBI mortality was $17 \%$. This would suggest that we are missing the opportunity to prevent or decrease secondary brain injury, and/or our ability to detect change in neurologic examination and intervene appropriately is suboptimal. This represents a significant opportunity for intervention.

Guidelines and protocols aimed at reducing secondary brain injury ${ }^{\text {I9 }}$ should be implemented and adhered to. Patients should first be resuscitated in accordance with Advanced Trauma Life Support guidelines, ${ }^{20}$ assessing the airway, breathing, and

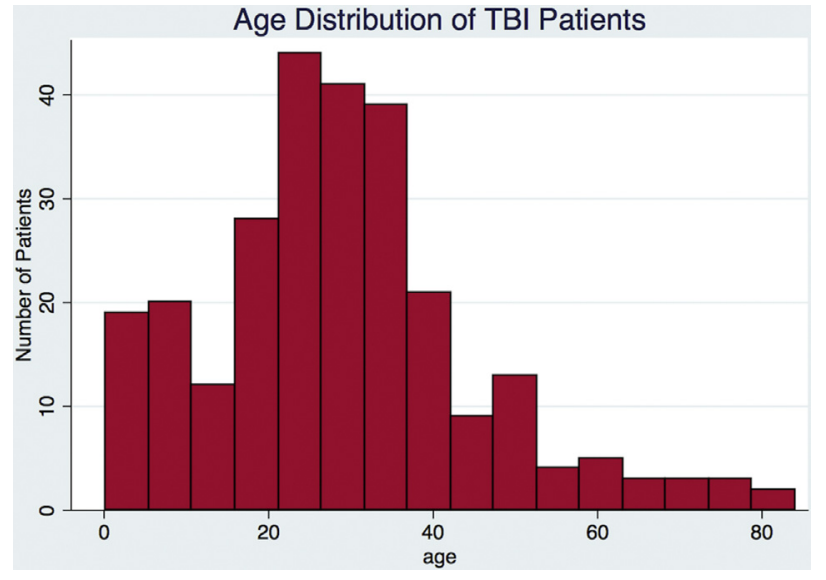

Figure 2. Age distribution of all patients admitted to Kamuzu Central Hospital with traumatic brain injury. TBI, traumatic brain injury.

circulation in turn. Supplementary oxygen is available in the casualty department, but it is not common practice to intubate patients, even those with GCS scores $\leq 8$, until they have been moved to the ICU. We found that only $27 \%$ of surviving patients with severe TBI were intubated to protect the airway at any time during their hospitalization. Keeping in mind that patients often have concomitant injuries, care should be taken to assess the entire patient, including the cervical spine. Only $14.3 \%$ of our patients received a neck collar at any time during their hospitalization. After patients are stabilized, it is generally recommended that any patient with a moderate to severe TBI undergo CT scanning. ${ }^{2 \mathrm{I}}$ In our setting, $48 \%$ of patients did not undergo CT scan.

Early protection of the airway, prompt CT scanning, and placement of neck collars on all trauma patients are all practices that should be improved on to optimize the care of TBI patients at our hospital. Additionally, I5\% of patients with severe TBI received care in the general ward, where staffing and monitoring is limited. This poses an additional challenge because patients in the general ward do not receive frequent neurologic checks, which are critical to prompt further interventions.

One major component of secondary brain injury prevention is the management of intracranial pressure (ICP), as a precipitous rise in ICP can quickly lead to herniation and brain death. Although invasive ICP monitoring is not possible in our setting, there is no evidence to suggest that invasive ICP monitoring reduces mortality in any setting. ${ }^{22}$ However, simple procedures aimed at maintaining a normal ICP, such as elevation of the head of the bed should be routine. ${ }^{2 \mathrm{I}}$ To prevent the devastating consequences of a sudden rise in ICP, frequent neurologic checks should be performed to detect clinical signs of increased ICP, such as a decrease in the patient's GCS score. If a sudden decrease in GCS score or the development of a lateralizing sign is detected, the patient may have an expanding extra-axial hematoma, requiring evacuation..$^{21}$ In these patients, outcome is markedly affected by the time to intervention, with hematomas requiring drainage within 4 hours to prevent significant morbidity and mortality. ${ }^{23}$ 
The standard care practices described here-timely placement of interventions such as neck collars, appropriate radiographic studies, referral of the patient to the correct level of care, and frequent neurologic checks to prompt further interventions as necessary-may all be addressed even in a resource-poor setting with the implementation of evidence-based management protocols, in which all TBI patients receive uniform multidisciplinary care based on guidelines. ${ }^{24}$ Studies have shown that the use of management protocols for patients with severe TBI are associated with reductions in death up to 6 months after hospital discharge, and overall improved neurologic outcome. ${ }^{25}$ Although management protocols would have to be contextualized to the setting, ${ }^{26}$ their implementation may make it possible to ensure that all patients receive the highest level of care possible.

Our study is limited by a lack of postdischarge follow-up because long-term outcomes could not be determined. We are also unable to determine the incidence of TBI in our population because of presentation bias. We missed those patients who died prior to arrival to the hospital or present only to the district hospitals.

\section{CONCLUSIONS}

Trauma is a significant contributor to global morbidity and mortality, and a growing public health concern, particularly in LMICs. The attenuation of TBI-related morbidity and mortality is critical to reduce the global impact of trauma. In our setting, significant mortality is seen even in patients with mild TBI. Contextualized management protocols may help attenuate TBI-related mortality and improve functional outcome in our lowresource setting.

\section{ACKNOWLEDGMENTS}

The authors wish to thank the Department of Surgery at Kamuzu Central Hospital and UNC-Project Malawi for their support of this work.

\section{REFERENCES}

I. GBD 2013 DALYs and HALE Collaborators, Murray CJ, Barber RM, Foreman KJ, Abbasoglu Ozgoren A, Abd-Allah F, et al. Global, regional and national disability-adjusted life years (DALYs) for 306 diseases and injuries and healthy-life expectance for I88 countries, 1990-2013: quantifying the epidemiological transition. Lancet. 2015; 386:2I45-2I9I.

2. Capone-Neto A, Rizoli SB. Linking the chain of survival: trauma as a traditional role model for multisystem trauma and brain injury. Curr Opin Crit Care. 2009;15:290-294.

3. de Ramirez SS, Hyder AA, Herbert HK, Stevens K. Unintentional injuries: magnitude, prevention, and control. Annu Rev Public Health. 2012;33:I75-I9I.

4. De Silva MJ, Roberts I, Perel P, Edwards P, Kenward MG, Fernandes J, et al. Patient outcome after traumatic brain injury in high-, middle- and low-income countries: analysis of data on 8927 patients in 46 countries. Int J Epidemiol. 2009;38: $452-458$.

5. Hyder AA, Wunderlich CA, Puvanachandra P, Guruaj G, Kobusingye OC. The impact of traumatic brain injuries: a global perspective. NeuroRehabilitation. 2007;22:34I-353.

6. Hode L, Madougou S, Fatigba HO, Hounnou P, Ebassa K, Hans Moevi AA, et al. The direct cost of treatment of traumatic brain injury in a SubSaharan African country (Benin). World Neurosurg. 2017;99:210-2I3.

7. Trading Economics. Malawi GDP per Capita. Available at: https://tradingeconomics.com/ malawi/gdp-per-capita. Accessed July 23, 2017.

8. Tyson AF, Varela C, Cairns BA, Charles AG. Hospital mortality following trauma: an analysis of a hospital-based injury surveillance registry in sub-Saharan Africa. J Surg Educ. 2015;72:e66-e72.

9. Lanlois JA, Rutland-Brown W, Wald MM. The epidemiology and impact of traumatic brain injury: a brief overview. J Head Trauma Rehabil. 2006;2I:375-378.
Io. Thurman DJ, Alverson C, Dunn KA, Guerrero J, Sniezek JE. Traumatic brain injury in the United States: a public health perspective. J Head Traum Rehabil. I999;14:602-615.

II. Wong JC, Linn KA, Shinohara RT, Mateen FJ. Traumatic brain injury in Africa in 2050: a modeling study. Eur J Neurol. 2016;23:382-386.

I2. Nell V, Digby SO. Epidemiology of traumatic brain injury in Johannesburg-II. Morbidity, mortality and etiology. Soc Sci Med. I991;33:289-296.

13. Kapapa T. Traumatic brain injury in Africa: The Republic of Malawi as an example. East Cent Afr J Surg. 2014;19:55-58.

I4. Maier D, Njoku I Jr, Schmutzhard E, Dharsee J, Doppler M, Härtl R, et al. Traumatic brain injury in a rural and an urban Tanzanian hospital-a comparative, retrospective analysis based on computed tomography. World Neurosurg. 20I4;8I: 478-482.

15. Tran TM, Fuller AT, Kiryabwire J, Mukasa J, Muhumuza M, Ssenyojo H, et al. Distribution and characteristics of severe traumatic brain injury at Mulago National Referral Hospital in Uganda. World Neurosurg. 2015;83:269-277.

I6. Udoh DO, Adeyemo AA. Traumatic brain injuries in children: a hospital-based study in Nigeria. Afr J Paediatr Surg. 2013;10:154-I59.

I7. Aenderl I, Gashaw T, Siebeck M, Mutschler W. Head injury-a neglected public health problem: a four-month prospective study at Jimma University Specialized Hospital, Ethiopia. Ethiop J Health Sci. 20I4;24:27.

I8. Kraus JF, Chu LC. Epidemiology. In: Silver JM, McCallister TW, Yudofsky SC, eds. Textbook of Traumatic Brain Injury. Vol. I. Washington, DC: American Psychiatric Publishing, Inc; 2005:3-26.

I9. Brain Trauma Foundation; American Association of Neurological Surgeons; Congress of Neurological Surgeons; Joint Section on Neurotrauma and Critical Care, AANS/CNS, Carney NA. Guidelines for the management of severe traumatic brain injury. Methods. J Neurotrauma. 2007;24:S3-S6.
20. ATLS Subcommittee; American College of Surgeons' Committee on Trauma; International ATLS Working Group. Advanced Trauma Life Support (ATLS): the ninth edition. J Trauma Acute Care Surg. 2013;74:1363-1366.

2I. Greenberg MS. Head trauma. In: Hiscock T, ed, Handbook of Neurosurgery. New York, NY: Thieme 2010:850-929.

22. Aiolfi A, Benjamin E, Khor D, Inaba K, Lam L, Demetriades D. Brain trauma foundation guidelines for intracranial pressure monitoring: compliance and effect on outcome. World J Surg. 2017;4I:I543-I549.

23. Seelig JM, Becker D, Miller D, Greenberg RP, Ward JD, Choi SC. Traumatic acute subdural hematoma: major mortality reduction in comatose patients treated with four hours. N Engl J Med. I98I;304:I5II-I5I8.

24. Helmy A, Vizcaychipi M, Gupta AK. Traumatic brain injury: intensive care management. $\mathrm{Br}$ Anaesth. 2007;99:32-42.

25. English SW, Turgeon AF, Owen E, Doucette S, Pagliarello G, McIntyre L. Protocol management of severe traumatic brain injury in intensive care units: a systematic review. Neurocrit Care. 2013;18:I3I-I42.

26. Mwita C, Muthoka J, Maina S, Mulingwa P, Gwer S. Early management of traumatic brain injury in a Tertiary hospital in Central Kenya: a clinical audit. J Neurosci Rural Pract. 2016;7:97.

Conflict of interest statement: The Fulbright Program supported this work and the National Institutes of Health through the Fogarty Global Health Fellows Program Consortium comprised of the University of North Carolina, John Hopkins, Morehouse, and Tulane (R25TW009340).

Received 7 July 2017; accepted 13 September 2017

Citation: World Neurosurg. (2017) 108:650-655. https://doi.org/10.1016/j.wneu.2017.09.084 Journal homepage: www.WORLDNEUROSURGERY.org 